\title{
Autoregulated and individualised resistance training versus pre-determined and standardised resistance training in tendinopathy: A systematic review protocol
}

Ian Burton MSc, CSCS

Physiotherapist, Angus HSCP, NHS Tayside, Dundee, United Kingdom

Please cite as: Burton, I. (2020). Autoregulated and individualised resistance training versus pre-determined and standardised resistance training in tendinopathy: A systematic review protocol.

SportRxiv doi: $10.31236 / o s f . i o / q g y 9 j$

\section{ABSTRACT}

Review Objective: To synthesize the best available evidence on the effectiveness of interventions that have used autoregulated and individualised resistance training versus pre-determined and standardised resistance training in treating any tendinopathy.

Introduction: Recent evidence suggests that individualised exercise may be more effective than standardised exercise for musculoskeletal disorders such as tendinopathy. However, no systematic reviews have been conducted on the topic and optimal treatment protocols and clinical recommendations are lacking. 
Inclusion criteria: Randomised controlled trials assessing the effectiveness of autoregulated and individualised resistance training versus pre-determined and standardised resistance training for tendinopathy in adults will be included.

Methods: The authors will search for a wide range of sources to find both published and unpublished studies via EBSCOhost, including, but not limited to, MEDLINE, SPORTDiscus, CINAHL,Cochrane Central Register of Controlled Trials (CENTRAL), and Allied and Complementary Medicine Database (AMED). Studies published in a language other than English will only be considered if a translation is available. The JBI systematic review methodology will be followed when conducting the review. Data synthesis will be conducted using meta-analysis or narrative synthesis, where appropriate.

\section{Systematic review registration number: CRD42020213400}

Keywords: Exercise; Resistance training; Autoregulation; Tendinopathy; Musculoskeletal Diseases

\section{Introduction}

Tendinopathy is a chronic musculoskeletal disorder, causing pain and decreased strength and physical function. Tendinopathy accounts for more than $30 \%$ of sports injuries and $7 \%$ of health practitioner visits. ${ }^{1}$ Due to their role in force transmission during loading, tendons are susceptible to large forces that can ultimately cause structural derangement and injury. ${ }^{2}$ The aetiology of tendinopathy is not fully understood; however, the condition is known to be derived from repetitive tendon microtrauma. ${ }^{3}$ The pathogenesis of tendinopathy involves a disrupted healing process that manifests as increased tendon thickness, neovascularization, damaged collagen fibres and incidence of tissue calcification. ${ }^{4}$ Although inflammatory cells may be present, the term tendinopathy is preferred to 'tendinitis' to reflect the often non-inflammatory and degenerative nature of the condition. ${ }^{5}$ 
Tendinopathies are challenging to treat and are considered to have a multifactorial pathogenesis resulting from a range of extrinsic and intrinsic factors. ${ }^{6}$ Recent European data on lower limb tendinopathies estimate incidence and prevalence ranging from 7.0-11.8 and 10.5-16.6 per 1000 people, respectively. ${ }^{7-8}$ Tendinopathy prevalence has been shown to be higher in athletes due to frequent jumping, landing, running and change of direction movements. ${ }^{9}$ Prevalence of plantar heel pain has been reported to be as high as $18 \%$ among runners. ${ }^{10}$ Whereas, prevalence of Achilles and patellar tendinopathy have been reported to be as high as 23 and $45 \%$ in runners and elite male volleyball players, respectively. ${ }^{11-12} \mathrm{~A}$ range of common approaches are used in the treatment of tendinopathy including exercise, corticosteroid injections, shockwave therapy, laser therapy, ultrasound, platelet-rich plasma injections and manual therapy. ${ }^{13}$ Recent evidence indicates that exercise may be the most effective therapy, particularly heavy load resistance training. ${ }^{14}$

Through the process of mechano-transduction, exercise can increase tissue regeneration by stimulating cellular responses and counteract structural adaptations of tendinopathy including disorganized collagen architecture. ${ }^{15}$ Loading forces applied to tendons during resistance training may also stimulate remodelling of abnormal tendon structure and improve the mechanical properties of the tendon. ${ }^{16-17}$ Heavy slow resistance training (HSRT) involving both concentric and eccentric strengthening, or heavy eccentric training alone, has been shown to produce positive outcomes for lower limb tendinopathies including Achilles, patellar, and plantar heel pain. ${ }^{18-20}$ The high forces and loads produced during HSRT and slow eccentric contractions may stimulate tendon remodelling and compliance by increasing collagen synthesis and reducing neovascularization when applied progressively. ${ }^{21}$ Eccentric loading has also been reported to be more effective than stretching for inducing length chances in short muscles, reducing injury risk and improving rehabilitation outcomes. ${ }^{22}$

Individual variability in factors such as genetics, sleep, nutrition, psychological stress, lifestyle factors and the total exercise stimulus experienced may affect performance during resistance training in rehabilitation. ${ }^{23}$ Pre-determined exercise 
programs including those prescribed during rehabilitation do not account for variation in individual response and as such may limit improvements compared to less stringent alternatives. ${ }^{24}$ Considering individual factors within rehabilitation and adjusting training to match increases in strength may improve effectiveness and prevent training plateaus. ${ }^{25-26}$ Autoregulation is a form of training which involves the participant self-adjusting the variables of resistance training based on their daily or weekly readiness to train and training response. ${ }^{25}$ Due to the multifactorial nature and wide variances in individual responses to training, it is suggested that autoregulation training may lead to improved training adaptations compared to traditional standardised training. ${ }^{25}$ Although autoregulation originated in physiotherapy research and is a common practice in powerlifting training there is a lack of current research on its effectiveness. ${ }^{25}$ Studies have shown that when exercise programs are tailored to the individual, training adaptations are enhanced. ${ }^{27}$ However, despite the increased use of resistance training protocols for tendinopathy and the well-known principles of individualisation and tailored rehabilitation, research studies frequently comprise pre-determined protocols that are not individually tailored or progressed. ${ }^{28}$

Despite increased awareness of the importance of identifying methods to enable self-management of exercise in physiotherapy, there appears to be a paucity of research investigating individualised progression methods. ${ }^{29}$ A common limitation of pre-determined exercise programs for tendinopathy is that prescription and progression of repetitions and sets are often poorly defined, and as a result desired training loads may not being achieved. ${ }^{30-31}$ If the exercise dosage is insufficient, then the mechanobiological stimulus may not be adequate to stimulate healing, resulting in poor outcomes. ${ }^{15}$ Previous studies have shown an association between increased exercise dose and recovery for musculoskeletal disorders. ${ }^{32}$ However, inappropriate monitoring and progression of the stimulus achieved with resistance training may be related to a higher risk of injury, suggesting better methods are required. ${ }^{33}$

Previous studies investigating tendinopathy rehabilitation have encouraged patients to monitor pain response whilst exercising. Whilst pain may be produced 
during exercise, a common recommendation that has been made is that the pain produced should be no worse upon cessation. ${ }^{34-35}$ Progression via pain-monitoring in Achilles tendinopathy was identified as a feasible method to regulate plyometric exercise such as sprinting, jumping and hopping. Silbernagel et al. 2007 demonstrated that by employing a pain monitoring model to adjust exercise, patients were able to improve function and pain during loading at a similar rate to patients assigned to active rest. ${ }^{36}$ However, other than pre-determined or painmonitoring progression methods in tendinopathy rehabilitation, no other progression or exercise tailoring method such as autoregulation of training, is widely identified in the literature or clinical practice.

A search in PROSPERO, The Cochrane Library and in PubMed was performed and identified no systematic reviews comparing the effectiveness of autoregulated and individualised resistance training versus pre-determined and standardised resistance training for tendinopathy. Therefore, the aim of this work is to conduct such a systematic review and if possible meta-analysis to synthesise the available evidence and inform recommendations regarding the optimal resistance training interventions for treating tendinopathy. This systematic review and meta-analysis will be conducted in accordance with the Joanna Briggs Institute (JBI) methodology for systematic reviews of effectiveness and will be conducted in accordance with the a priori protocol presented here and registered in the PROSPERO database (CRD42020213400). ${ }^{37}$

\section{Review question}

What is the effectiveness of autoregulated and individualised resistance training versus pre-determined and standardised resistance training in treating tendinopathies?

\section{Inclusion Criteria}

\section{Participants}


This review will consider studies with adult participants aged 18 years old or over, clinically diagnosed with a tendinopathy.

\section{Interventions}

This review will include studies that have investigated the effectiveness of autoregulated and individualised resistance training versus pre-determined and standardised resistance training in treating tendinopathies. Resistance training can include strengthening exercises, heavy slow resistance training, plyometric, or combined exercise interventions. Any home or healthcare setting including physiotherapy or podiatry clinics and departments, outpatient departments, primary care settings, specialist orthopaedic or surgical clinics, and rehabilitation clinics will be permitted.

\section{Comparators}

Any pre-determined and standardised resistance training interventions, including eccentric, concentric, heavy slow resistance training and interventions using combined types of resistance training. Trials with two or more arms will be included. The use of active co-interventions such as pain medication (NSAIDs), education, orthotics and stretching exercise will be accepted if used in all arms of the trial.

\section{Outcomes}

Primary outcomes will include pain and function. Pain evaluated by any validated scale such as the Visual Analogue Scale (VAS), Numeric Rating Scale (NRS), verbal rating scales or Foot Function Index pain subscale (FFI-PS) will be included. ${ }^{38}$ Function evaluated by any validated scale for tendinopathies such as the Foot Function Index (FFI), Foot and Ankle Ability Measure (FAAM), Victoria Institute of Sport Assessment Achilles questionnaire (VISA-A), Victoria Institute of Sport Assessment Patella questionnaire (VISA-P), Disabilities of the Arm, Shoulder and Hand (DASH). ${ }^{39-40}$ Additional secondary outcomes that will be considered for 
inclusion if available include quality of life measures using validated scales such as the EQ-5D-5L and global rating of change (GRoC) scores.

\section{Types of studies}

The review will be restricted to randomized controlled trials (RCTs) in which autoregulated and individualised resistance training was compared with predetermined and standardised resistance training in treating any tendinopathy. Trials with two or more arms will be considered for inclusion. The use of active cointerventions such as pain medication (NSAIDs), education, orthotics and exercise will be acceptable if used in all trial arms, to limit confounding. ${ }^{41}$ In the hierarchy of evidence, systematic reviews of RCTs offer the highest level of evidence. ${ }^{42}$ The strongest inferences can be drawn if the review is well conducted and includes methodologically sound RCTs with consistent results. ${ }^{43}$ The authors preliminary work has identified several potentially eligible RCTs. Therefore, due to the availability of RCTs on this topic, they will be chosen for inclusion over less robust study designs. Any deviation from the standard RCT design such as crossover or cluster designed trials will also be included.

\section{Methods}

The systematic review process will follow the Preferred Reporting Items for Systematic Reviews and Meta-Analyses (PRISMA) and JBI systematic reviews of effectiveness guidelines. ${ }^{37}$ The review has been registered in the PROSPERO database (CRD42020213400).

\section{Search strategy}

The search strategy will seek to identify published and unpublished trials utilizing a three-step search strategy. An initial scoping search of Medline will be conducted, followed by analysis of text words contained in the title and abstract and article index terms. A comprehensive systematic search using all identified keywords and index terms will then be conducted using the following databases: Medline, CINAHL, AMED, SPORTDiscus, PEDro, Cochrane CENTRAL. The search for 
unpublished studies will include EThOS Networked Digital Library of Theses and Dissertations and Google Scholar. The trial registers to be searched include: ClinicalTrials.gov, UK clinical trials gateway, EU trials registry. Finally, in addition to the comprehensive search, supplementary searches will be undertaken from reviewing bibliographies of articles selected for critical appraisal and related systematic reviews to find those not initially identified. Databases will be searched from 1998 to 2020. The year 1998 will be used to ensure seminal work is not missed as clinical interventional research into resistance training for tendinopathy was first published in 1998 with the Alfredson protocol for Achilles tendinopathy. Studies published in a language other than English will only be considered if a translation is available as translation services are not available to the authors.

\section{Study selection}

All identified citations from the systematic search will be uploaded into RefWorks (Proquest LLC), with duplicates removed. Two reviewers will independently screen the titles and abstracts of all studies obtained against the identified inclusion criteria. Full-text versions of eligible studies will be accessed and reviewed against the inclusion criteria. Studies will be removed from the screening process if the information provided does not meet the criteria. The details of studies meeting the criteria will be imported in to Covidence. ${ }^{37}$

\section{Assessment of methodological quality}

Included studies will be critically appraised by two independent reviewers at study level for methodological quality in the review using the standardized Cochrane risk of bias tool on Covidence. ${ }^{37}$ Any disagreements that arise between the reviewers will be resolved through discussion or with a third reviewer. The results of critical appraisal will be reported in narrative form, and in a table. The critical appraisal results will be presented in a table and narrative form. All studies meeting inclusion criteria, regardless of their methodological quality, will undergo data extraction and synthesis and be included in the review. 


\section{Data extraction}

Data will be extracted from papers included in the review using the standardised data extraction tool available on Covidence ${ }^{37}$ by two independent reviewers. The data extracted will include specific details relative to the interventions, comparators, populations, study methods and outcomes of significance to the review question which include heel pain and foot function. Any disagreements that arise between the reviewers will be resolved through discussion or with a third reviewer. Authors of papers will be contacted to request missing or additional data, where required.

\section{Data synthesis}

Quantitative data will, where possible be pooled in statistical meta-analysis using RevMan software. All results will be subject to double data entry. Effect sizes expressed as odds ratio (for categorical data) and weighted mean differences (for continuous data) and their $95 \%$ confidence intervals will be calculated for analysis. Heterogeneity will be assessed statistically using the standard $x^{2}$ and explored using subgroup analyses if possible based on the different quantitative study designs included in this review. Where statistical pooling is not possible due to heterogeneity, the findings will be presented in narrative form including tables and figures to aid in data presentation where appropriate. Analysis of subgroups or subsets is not planned, although the sources of any heterogeneity detected will be explored using subgroup analyses based on the different quantitative study designs included in the review.

\section{Assessing certainty in the findings}

A 'summary of findings' table will be created following The Grading of Recommendations, Assessment, Development and Evaluation (GRADE) approach for assessing the quality of evidence. ${ }^{44}$ Evidence from RCTs starts at high quality and the certainty is increased or decreased for several reasons, such as risk of bias. ${ }^{45}$ The outcomes reported in the summary of findings table will include pain and function for the interventions. For each outcome, a ranking 
of 'high', 'moderate', 'low' or 'very low' will be assigned to the quality of evidence based on the risk of bias. There is by necessity a considerable amount of subjectivity in each decision as GRADE cannot be implemented mechanically. However, GRADE does provide a reproducible and transparent framework for grading certainty in evidence. ${ }^{46}$

\section{Acknowledgments}

None.

\section{Funding}

No sources of funding will contribute to this systematic review.

\section{Conflicts of interest}

The authors declare no conflict of interest. 


\section{References}

1. Skjong CC, Meininger AK, Ho SS. Tendinopathy treatment: where is the evidence? Clin Sports Med 2012 Apr;31(2):329-350.

2. Magnusson SP, Langberg $H$, Kjaer $M$. The pathogenesis of tendinopathy: balancing the response to loading. Nat Rev Rheumatol 2010 May;6(5):262-268.

3. Fu SC, Rolf C, Cheuk YC, Lui PP, Chan KM. Deciphering the pathogenesis of tendinopathy: a three-stages process. Sports Med Arthrosc Rehabil Ther Technol 2010 Dec 13;2:30-2555-2-30.

4. Fredberg U, Stengaard-Pedersen K. Chronic tendinopathy tissue pathology, pain mechanisms, and etiology with a special focus on inflammation. Scand J Med Sci Sports 2008 Feb;18(1):3-15.

5. Rees JD, Stride M, Scott A. Tendons--time to revisit inflammation. Br J Sports Med 2014 Nov;48(21):1553-1557.

6. Seitz AL, McClure PW, Finucane S, Boardman ND,3rd, Michener LA. Mechanisms of rotator cuff tendinopathy: intrinsic, extrinsic, or both? Clin Biomech (Bristol, Avon) 2011 Jan;26(1):1-12.

7. Albers IS, Zwerver J, Diercks RL, Dekker JH, Van den Akker-Scheek I. Incidence and prevalence of lower extremity tendinopathy in a Dutch general practice population: a cross sectional study. BMC Musculoskelet Disord 2016 Jan 13;17:16016-0885-2.

8. Riel H, Lindstrom CF, Rathleff MS, Jensen MB, Olesen JL. Prevalence and incidence rate of lower-extremity tendinopathies in a Danish general practice: a registry-based study. BMC Musculoskelet Disord 2019 May 22;20(1):239-0192629-6.

9. Zwerver J, Bredeweg SW, van den Akker-Scheek I. Prevalence of Jumper's knee among nonelite athletes from different sports: a cross-sectional survey. Am J Sports Med 2011 Sep;39(9):1984-1988. 
10. Janssen I, van der Worp $H$, Hensing S, Zwerver J. Investigating Achilles and patellar tendinopathy prevalence in elite athletics. Res Sports Med 2018 JanMar;26(1):1-12.

11. Sprague AL, Smith AH, Knox P, Pohlig RT, Gravare Silbernagel K. Modifiable risk factors for patellar tendinopathy in athletes: a systematic review and metaanalysis. Br J Sports Med 2018 Dec;52(24):1575-1585.

12. Arnold MJ, Moody AL. Common Running Injuries: Evaluation and Management. Am Fam Physician 2018 Apr 15;97(8):510-516.

13. Cardoso TB, Pizzari T, Kinsella R, Hope D, Cook JL. Current trends in tendinopathy management. Best Pract Res Clin Rheumatol 2019 Feb;33(1):122140.

14. Malliaras $\mathrm{P}$, Barton $\mathrm{CJ}$, Reeves ND, Langberg $\mathrm{H}$. Achilles and patellar tendinopathy loading programmes : a systematic review comparing clinical outcomes and identifying potential mechanisms for effectiveness. Sports Med 2013 Apr;43(4):267-286.

15. Khan KM, Scott A. Mechanotherapy: how physical therapists' prescription of exercise promotes tissue repair. Br J Sports Med 2009 Apr;43(4):247-252.

16. Wang JH, Guo Q, Li B. Tendon biomechanics and mechanobiology--a minireview of basic concepts and recent advancements. J Hand Ther 2012 AprJun;25(2):133-40; quiz 141.

17. Bohm S, Mersmann F, Arampatzis A. Human tendon adaptation in response to mechanical loading: a systematic review and meta-analysis of exercise intervention studies on healthy adults. Sports Med Open 2015 Dec;1(1):7-0150009-9. Epub 2015 Mar 27.

18. Beyer R, Kongsgaard M, Hougs Kjaer B, Ohlenschlaeger T, Kjaer M, Magnusson SP. Heavy Slow Resistance Versus Eccentric Training as Treatment for Achilles Tendinopathy: A Randomized Controlled Trial. Am J Sports Med 2015 Jul;43(7):1704-1711. 
19. Kongsgaard M, Kovanen V, Aagaard P, Doessing S, Hansen P, Laursen AH, et al. Corticosteroid injections, eccentric decline squat training and heavy slow resistance training in patellar tendinopathy. Scand J Med Sci Sports 2009 Dec;19(6):790-802.

20. Rathleff MS, Molgaard CM, Fredberg U, Kaalund S, Andersen KB, Jensen TT, et al. High-load strength training improves outcome in patients with plantar fasciitis: A randomized controlled trial with 12-month follow-up. Scand J Med Sci Sports 2015 Jun;25(3):e292-300.

21. Murtaugh B, Ihm JM. Eccentric training for the treatment of tendinopathies. Curr Sports Med Rep 2013 May-Jun;12(3):175-182.

22. Timmins RG, Ruddy JD, Presland J, Maniar N, Shield AJ, Williams MD, et al. Architectural Changes of the Biceps Femoris Long Head after Concentric or Eccentric Training. Med Sci Sports Exerc 2016 Mar;48(3):499-508.

23. Mann TN, Lamberts RP, Lambert MI. High responders and low responders: factors associated with individual variation in response to standardized training. Sports Med 2014 Aug;44(8):1113-1124.

24. Timmons JA. Variability in training-induced skeletal muscle adaptation. J Appl Physiol (1985) 2011 Mar;110(3):846-853.

25. Mann JB, Thyfault JP, Ivey PA, Sayers SP. The effect of autoregulatory progressive resistance exercise vs. linear periodization on strength improvement in college athletes. J Strength Cond Res 2010 Jul;24(7):1718-1723.

26. Kiely J. Periodization Theory: Confronting an Inconvenient Truth. Sports Med 2018 Apr;48(4):753-764.

27. Jones N, Kiely J, Suraci B, Collins DJ, de Lorenzo D, Pickering C, et al. A genetic-based algorithm for personalized resistance training. Biol Sport 2016 Jun;33(2):117-126.

28. Ciolac EG, Rodrigues-da-Silva JM. Resistance Training as a Tool for Preventing and Treating Musculoskeletal Disorders. Sports Med 2016 Sep;46(9):1239-1248. 
29. Peek K, Sanson-Fisher R, Mackenzie L, Carey M. Interventions to aid patient adherence to physiotherapist prescribed self-management strategies: a systematic review. Physiotherapy 2016 Jun;102(2):127-135.

30. Couppe C, Svensson RB, Silbernagel KG, Langberg H, Magnusson SP. Eccentric or Concentric Exercises for the Treatment of Tendinopathies? J Orthop Sports Phys Ther 2015 Nov;45(11):853-863.

31. Riel H, Jensen MB, Olesen JL, Vicenzino B, Rathleff MS. Self-dosed and predetermined progressive heavy-slow resistance training have similar effects in people with plantar fasciopathy: a randomised trial. J Physiother 2019 Jul;65(3):144-151.

32. Osteras B, Osteras $H$, Torstensen TA, Vasseljen O. Dose-response effects of medical exercise therapy in patients with patellofemoral pain syndrome: a randomised controlled clinical trial. Physiotherapy 2013 Jun;99(2):126-131.

33. Gabbett TJ. The training-injury prevention paradox: should athletes be training smarter and harder? Br J Sports Med 2016 Mar;50(5):273-280.

34. Littlewood C, Ashton J, Mawson S, May S, Walters S. A mixed methods study to evaluate the clinical and cost-effectiveness of a self-managed exercise programme versus usual physiotherapy for chronic rotator cuff disorders: protocol for the SELF study. BMC Musculoskelet Disord 2012 Apr 30;13:62-2474-13-62.

35. Littlewood C, Bateman M, Brown K, Bury J, Mawson S, May S, et al. A selfmanaged single exercise programme versus usual physiotherapy treatment for rotator cuff tendinopathy: a randomised controlled trial (the SELF study). Clin Rehabil 2016 Jul;30(7):686-696.

36. Silbernagel KG, Thomee R, Eriksson BI, Karlsson J. Continued sports activity, using a pain-monitoring model, during rehabilitation in patients with Achilles tendinopathy: a randomized controlled study. Am J Sports Med 2007 Jun;35(6):897-906.

37. Tufanaru C, Munn Z, Aromataris E, Campbell J, Hopp L. Chapter 3: Systematic reviews of effectiveness. In: Aromataris E, Munn Z (Editors). Joanna Briggs 
Institute Reviewer's Manual. The Joanna Briggs Institute, 2017. Available from https://reviewersmanual.joannabriggs.org/

38. Boonstra AM, Schiphorst Preuper HR, Reneman MF, Posthumus JB, Stewart RE. Reliability and validity of the visual analogue scale for disability in patients with chronic musculoskeletal pain. Int J Rehabil Res 2008 Jun;31(2):165-169.

39. Budiman-Mak E, Conrad K, Stuck R, Matters M. Theoretical model and Rasch analysis to develop a revised Foot Function Index. Foot Ankle Int 2006 Jul;27(7):519-527.

40. Budiman-Mak E, Conrad KJ, Mazza J, Stuck RM. A review of the foot function index and the foot function index - revised. J Foot Ankle Res 2013 Feb 1;6(1):51146-6-5.

41. Arah OA. Bias Analysis for Uncontrolled Confounding in the Health Sciences. Annu Rev Public Health 2017 Mar 20;38:23-38.

42. Guyatt GH, Oxman AD, Kunz R, Jaeschke R, Helfand M, Liberati $A$, et al. Incorporating considerations of resources use into grading recommendations. $B M]$ 2008 May 24;336(7654):1170-1173.

43. Charrois TL. Systematic reviews: what do you need to know to get started? Can J Hosp Pharm 2015 Mar-Apr;68(2):144-148.

44. Balshem $H$, Helfand M, Schunemann HJ, Oxman AD, Kunz R, Brozek J, et al. GRADE guidelines: 3. Rating the quality of evidence. J Clin Epidemiol 2011 Apr;64(4):401-406.

45. Sterne JA, Hernan MA, Reeves BC, Savovic J, Berkman ND, Viswanathan M, et al. ROBINS-I: a tool for assessing risk of bias in non-randomised studies of interventions. BMJ 2016 Oct 12;355:i4919.

46. Mustafa RA, Santesso N, Brozek J, Akl EA, Walter SD, Norman G, et al. The GRADE approach is reproducible in assessing the quality of evidence of quantitative evidence syntheses. J Clin Epidemiol 2013 Jul;66(7):736-42; quiz 742.e1-5. 